\title{
GENERALISATIONS OF UNIFORMLY NORMAL FAMILIES
}

\author{
W.K. HAYMAN AND A. HINKKANEN \\ In memory of Noel Baker
}

\begin{abstract}
We obtain separation and growth results for meromorphic functions $f$ in the unit disk such that for some positive integer $k, f \neq 0$ and $f^{(k)} \neq 1$ in the disk, or such that $f^{\prime} f^{k} \neq 1$ in the disk. These results are only slightly weaker than those for functions $f$ such that $|f|>\delta$ in the disk.
\end{abstract}

\section{INTRODUCTION}

In this paper, we prove the following result. We denote the unit disk by $\mathbb{D}$.

Theorem 1. Suppose that $f$ is analytic in $\mathbb{D}$ and that there exist positive functions $\delta(r)$ and $\lambda(r)$ defined in $(0,1)$ such that $\delta(r)$ decreases, $0<\delta(r)<1, \lambda(r)$ increases, and for all $r, 0<r<1$, the conditions

$$
\left|z_{1}\right| \leq r, \quad\left|z_{2}\right| \leq r
$$

and

$$
\left|f\left(z_{1}\right)\right| \leq e^{-\lambda(r)}, \quad\left|f\left(z_{2}\right)\right| \geq e^{\lambda(r)}
$$

imply that

$$
\left|\frac{z_{2}-z_{1}}{1-\overline{z_{1}} z_{2}}\right| \geq \delta(r)
$$

Then if $\left|z_{0}\right|<r$ and

$$
\log \left|f\left(z_{0}\right)\right|>\frac{8 \lambda(r)}{\delta(r)}
$$

AMS (2000) Mathematics Subject Classification. Primary 30D45. Secondary $30 \mathrm{D} 35$.

This material is based upon work supported by the National Science Foundation under Grant No. 0200752. 
we have

$$
\left|f^{\prime}\left(z_{0}\right)\right|<\frac{2 r\left|f\left(z_{0}\right)\right|}{r^{2}-\left|z_{0}\right|^{2}}\left\{\log \left|f\left(z_{0}\right)\right|+\frac{8 \lambda(r)}{\delta(r)}\right\} .
$$

If $\lambda(r)$ and $\delta(r)$ are constant, then the functions $f$ satisfying the hypotheses of Theorem 1 form a uniformly normal family in the sense of $[1]$.

In most of the applications we have

$$
\frac{8 \lambda(r)}{\delta(r)}=a+b \log \frac{1}{1-r}
$$

where $a$ and $b$ are non-negative constants.

Corollary 1. Let $f, \lambda(r)$ and $\delta(r)$ satisfy the hypotheses of Theorem 1 , and also (6). If $|z|=t$ and $r=1-\frac{1}{2}(1-t)^{2}$, then either $\log |f(z)| \leq \lambda^{*}(t)$ or

$$
\frac{\left|f^{\prime}(z)\right|}{|f(z)|}<\left\{\frac{2}{1-t^{2}}+8\right\}\left\{\log |f(z)|+\lambda^{*}(t)\right\},
$$

where $\lambda^{*}(t)=a+b \log 2+2 b \log \frac{1}{1-t}=a+b \log \frac{1}{1-r}$. Further we have in all cases

$$
\log |f(z)| \leq e^{8}\left\{3 a+8 b+\log ^{+}|f(0)|\right\} \frac{1+|z|}{1-|z|}
$$

\section{A SPECIAL CASE}

In this section we deal with the case when $\delta(r)$ and $\lambda(r)$ are constant.

Lemma 1. Suppose that $\lambda(r)=\lambda$ and $\delta(r)=\delta$, where $\lambda$ and $\delta$ are constants which satisfy the hypotheses of Theorem 1, and that (4) holds so that

$$
\alpha=\log \left|f\left(z_{0}\right)\right|>\frac{8 \lambda}{\delta}
$$

Then

$$
|f(z)|>e^{\lambda}, \quad \text { when }\left|\frac{z-z_{0}}{1-\overline{z_{0}} z}\right|<r_{1}
$$

where

$$
1-r_{1}=\frac{4 \lambda}{\alpha \delta}<\frac{1}{2}
$$


Thus

$$
\left|f^{\prime}\left(z_{0}\right)\right|<\frac{2\left|f\left(z_{0}\right)\right|}{1-\left|z_{0}\right|^{2}}\left\{\log \left|f\left(z_{0}\right)\right|+\frac{8 \lambda}{\delta}\right\}
$$

We proceed to prove (10) subject to (11). Since the hypotheses (1) to (4) are now conformally invariant, we assume without loss of generality that $z_{0}=0$. Let $r_{2}$ be maximal subject to $|f(z)|>e^{-\lambda}$ if $|z|<r_{2}$.

We suppose first that $r_{2}<1$. Let $r_{1}$ be the largest number such that

$$
|f(z)|>e^{\lambda} \quad \text { for }|z|<r_{1} .
$$

Then $0<r_{1}<r_{2}<1$ and there exists $z_{2}=r_{2} e^{i \theta}$ such that

$$
\left|f\left(z_{2}\right)\right|=e^{-\lambda} \text {. }
$$

We set $z_{1}=r_{1} e^{i \theta}$. Then (3) yields

$$
\left|\frac{z_{2}-z_{1}}{1-\overline{z_{1}} z_{2}}\right|=\frac{r_{2}-r_{1}}{1-r_{1} r_{2}} \geq \delta
$$

i.e.,

$$
r_{2} \geq \frac{r_{1}+\delta}{1+\delta r_{1}}
$$

We now apply Harnack's inequality to $\log |\phi|$ where

$$
\phi(z)=e^{\lambda} f\left(r_{2} z\right)
$$

and choose $z$ so that $|z|=r_{1} / r_{2}$ and $\left|f\left(r_{2} z\right)\right|=e^{\lambda}$. This is possible since $r_{1}$ is maximal subject to (13). Then $|\phi(z)|>1$ for $|z|<1$, and so

$$
\log |\phi(z)| \geq \frac{1-|z|}{1+|z|} \log |\phi(0)|
$$

i.e.,

$$
2 \lambda \geq \frac{r_{2}-r_{1}}{r_{2}+r_{1}}(\lambda+\alpha)
$$

If $r_{2}=1$ and $r_{1}$ is maximal subject to (13), then either $r_{1}=1$, in which case (14) and (16) are trivial, or we can choose $z$, such that $|z|=r_{1}$ and $\left|f\left(z_{1}\right)\right|=e^{\lambda}$. Now (15) still holds and we obtain (16) as before with $r_{2}=1$. Thus (16) is always true. Also (14) is true if $r_{2}=1$ and $r_{1}<1$, since $\delta<1$. Thus (14) and (16) always hold.

We substitute $r_{2}$ from (14) in (16) and obtain

$$
\begin{aligned}
\frac{2 \lambda}{\lambda+\alpha} & \geq\left(\frac{r_{1}+\delta}{1+\delta r_{1}}-r_{1}\right) /\left(\frac{r_{1}+\delta}{1+\delta r_{1}}+r_{1}\right)=\frac{\delta\left(1-r_{1}^{2}\right)}{\delta\left(1+r_{1}^{2}\right)+2 r_{1}} \\
& \geq \frac{\delta\left(1-r_{1}^{2}\right)}{\left(1+r_{1}\right)^{2}}=\delta \frac{1-r_{1}}{1+r_{1}} \geq \frac{\delta}{2}\left(1-r_{1}\right) .
\end{aligned}
$$


Thus

$$
1-r_{1} \leq \frac{4 \lambda}{\delta(\lambda+\alpha)} \leq \frac{4 \lambda}{\delta \alpha}<\frac{1}{2}
$$

by (9). This proves (10) subject to (11).

The function

$$
\Psi(z)=e^{-\lambda} f\left(r_{1} z\right)
$$

satisfies $|\Psi(z)|>1$ if $|z|<1$. Thus by Borel's inequality

$$
\left|\Psi^{\prime}(0)\right| \leq 2|\Psi(0)| \log |\Psi(0)|,
$$

i.e.,

$$
\begin{aligned}
\frac{\left|f^{\prime}(0)\right|}{|f(0)|} & \leq \frac{2}{r_{1}}(\log |f(0)|-\lambda)=\frac{2}{r_{1}}(\alpha-\lambda) \leq 2(\alpha-\lambda) \frac{1}{1-\frac{4 \lambda}{\delta \alpha}} \\
& =2(\alpha-\lambda)+2(\alpha-\lambda) \frac{4 \lambda}{\delta \alpha-4 \lambda} \\
& <2 \alpha+\frac{16 \alpha \lambda}{\delta \alpha}=2\left(\alpha+\frac{8 \lambda}{\delta}\right)
\end{aligned}
$$

since $\delta \alpha-4 \lambda>\delta \alpha / 2$ by (11). This proves (12) if $z_{0}=0$. If $z_{0} \neq 0$, we apply the above result to $F$ instead of $f$ at the origin, where $F(z)=$ $f\left(\left(z+z_{0}\right) /\left(1+\overline{z_{0}} z\right)\right)$. This yields (12) in general.

\section{Proof of Theorem 1}

To prove Theorem 1, we fix $r$ with $0<r<1$, and apply Lemma 1 with $F(z)=f(r z)$ instead of $f(z)$ and with $\lambda=\lambda(r)$ and $\delta=\delta(r)$.

We write $z_{1}=r Z_{1}, z_{2}=r Z_{2}$ and suppose that (1) and (2) imply (3). Thus if $\left|Z_{1}\right|<1,\left|Z_{2}\right|<1$ and

$$
\left|F\left(Z_{1}\right)\right| \leq e^{-\lambda}, \quad\left|F\left(Z_{2}\right)\right| \geq e^{\lambda}
$$

we deduce that

$$
\left|\frac{r\left(Z_{2}-Z_{1}\right)}{1-r^{2} \overline{Z_{1}} Z_{2}}\right| \geq \delta,
$$

with $\delta=\delta(r)$ and $\lambda=\lambda(r)$.

We next prove that

$$
\frac{\left|Z_{2}-Z_{1}\right|}{\left|1-\overline{Z_{1}} Z_{2}\right|}>\frac{r\left|Z_{2}-Z_{1}\right|}{\left|1-r^{2} \bar{Z}_{1} Z_{2}\right|} .
$$

To see this, note that $Z_{1} \neq Z_{2}$ and

$$
\left|1-r^{2} \overline{Z_{1}} Z_{2}\right|^{2}-r^{2}\left|1-\overline{Z_{1}} Z_{2}\right|^{2}=\left(1-r^{2}\right)\left(1-r^{2}\left|Z_{1}\right|^{2}\left|Z_{2}\right|^{2}\right)>0,
$$


which yields (19). Thus (18) implies that

$$
\left|\frac{Z_{2}-Z_{1}}{1-\overline{Z_{1}} Z_{2}}\right|>\delta
$$

So we can apply Lemma 1 to $F(z)=f(r z)$ instead of $f(z)$, and with $Z_{0}=z_{0} / r$ instead of $z_{0}$. This yields (5).

We next prove Corollary 1 . We have, with the notation of Corollary 1 ,

$$
\frac{1}{r^{2}-t^{2}}-\frac{1}{1-t^{2}}=\frac{(1-r)(1+r)}{(r-t)(r+t)(1-t)(1+t)} \leq \frac{(1-t)^{2}}{\frac{1}{2}(1-t)^{2} \cdot \frac{1}{2}}=4,
$$

since $r=1-\frac{1}{2}(1-t)^{2} \geq \frac{1}{2}$ and $r-t \geq \frac{1}{2}(1-t)$.

Thus (5) yields, with $\alpha=\log \left|f\left(z_{0}\right)\right|$,

$$
\left|f^{\prime}\left(z_{0}\right)\right|<\frac{2\left|f\left(z_{0}\right)\right|}{r^{2}-t^{2}}\left\{\alpha+\lambda^{*}(t)\right\}<2\left|f\left(z_{0}\right)\right|\left\{\frac{1}{1-t^{2}}+4\right\}\left\{\alpha+\lambda^{*}(t)\right\} .
$$

This proves $(7)$.

To deduce (8), we fix $\theta$ with $\theta \in[0,2 \pi)$ and write $y(t)=\log \left|f\left(t e^{i \theta}\right)\right|$ for $0 \leq t<1$. If $y(t) \leq \lambda^{*}(t)$ or if $t=0$, (8) clearly holds for $z=t e^{i \theta}$. So we suppose that for some $t$ with $0<t<1$, we have

$$
y(t)>\lambda^{*}(t) .
$$

We choose $t_{0}$ to be maximal, subject to $0 \leq t_{0}<t$ and $y\left(t_{0}\right) \leq \lambda^{*}\left(t_{0}\right)$. If $y(\tau)>\lambda^{*}(\tau)$ for all $\tau$ with $0 \leq \tau<t$, we set $t_{0}=0$. Then

$$
y(\tau)>\lambda^{*}(\tau) \text { for } t_{0}<\tau<t .
$$

Thus we can apply (7) with $\tau$ instead of $t$ in this range and obtain $y^{\prime}(\tau)-\left\{\frac{2}{1-\tau^{2}}+8\right\} y(\tau)<\left\{\frac{2}{1-\tau^{2}}+8\right\} \lambda^{*}(\tau), \quad$ for $t_{0}<\tau<t$.

Multiplying by

$$
P(\tau)=e^{-8 \tau} \frac{1-\tau}{1+\tau}
$$

and integrating with respect to $\tau$ from $t_{0}$ to $t$ we obtain

$$
y(t) \leq \frac{1}{P(t)}\left\{y\left(t_{0}\right) P\left(t_{0}\right)+\int_{t_{0}}^{t}\left(\frac{2}{1-\tau^{2}}+8\right) P(\tau) \lambda^{*}(\tau) d \tau\right\} .
$$

If $t_{0}=0$, we get $y\left(t_{0}\right) P\left(t_{0}\right)=y\left(t_{0}\right) \leq \log ^{+}|f(0)|$.

If $t_{0}>0$, we have $y\left(t_{0}\right) P\left(t_{0}\right)=\lambda^{*}\left(t_{0}\right) P\left(t_{0}\right)$.

We write

$$
\lambda^{*}(t)=a^{*}+b^{*} \log \frac{1}{1-t}
$$


with $a^{*}=a+b \log 2$ and $b^{*}=2 b$. Then

$$
\begin{aligned}
\lambda^{*}(t) P(t) & =\left(a^{*}+b^{*} \log \frac{1}{1-t}\right) \frac{1-t}{1+t} e^{-8 t} \\
& \leq a^{*}+b^{*} \sup _{0 \leq t<1}(1-t) \log \frac{1}{1-t}=a^{*}+\frac{b^{*}}{e} .
\end{aligned}
$$

Again

$$
\int_{0}^{1}\left(\frac{2}{1-t^{2}}+8\right) \frac{1-t}{1+t} e^{-8 t} d t<\int_{0}^{1} 10 e^{-8 t} d t<\frac{5}{4}
$$

while

$$
\begin{aligned}
& \int_{0}^{1}\left(\frac{2}{1-t^{2}}+8\right)\left(\frac{1-t}{1+t} \log \frac{1}{1-t}\right) e^{-8 t} d t \\
< & 2 \int_{0}^{1} \log \frac{1}{1-t} d t+\frac{8}{e} \int_{0}^{1} e^{-8 t} d t<2+\frac{1}{e} .
\end{aligned}
$$

Thus

$$
\int_{0}^{1}\left(\frac{2}{1-t^{2}}+8\right) P(t) \lambda^{*}(t) d t \leq \frac{5}{4} a^{*}+\left(2+\frac{1}{e}\right) b^{*} .
$$

Hence (21) yields finally

$$
\begin{aligned}
y(t) & \leq \frac{1}{P(t)}\left\{a^{*}+\frac{b^{*}}{e}+\frac{5}{4} a^{*}+\left(2+\frac{1}{e}\right) b^{*}+\log ^{+}|f(0)|\right\} \\
& \leq \frac{1}{P(t)}\left\{3 a+8 b+\log ^{+}|f(0)|\right\}
\end{aligned}
$$

which implies (8).

This proves Corollary 1.

\section{Applications}

Suppose that $M_{k}$ and $A_{k}$ are respectively the families of meromorphic functions $f$ in $\mathbb{D}$ and analytic functions $f$ in $\mathbb{D}$, such that $f(z) \neq 0$ and $f^{(k)}(z) \neq 1$ for all $z \in \mathbb{D}$. Then $M_{k}$ and in particular $A_{k}$ are normal families in $\mathbb{D}$ when $k \geq 1$, see, e.g., [3, Corollary 4.5.9, p. 150]. It follows that there exist positive constants $\lambda_{k}$ and $\delta_{k}$ depending only on $k$ such that if $f \in M_{k}$ and $\left|z_{j}\right| \leq \delta_{k}$, for $j=1,2$, then we cannot have $\left|f\left(z_{1}\right)\right| \leq e^{-\lambda_{k}}$ and $\left|f\left(z_{2}\right)\right| \geq e^{\lambda_{k}}$. In fact the conclusion must hold for every sufficiently small $\delta_{k}$, and $\lambda_{k}$ depending on $\delta_{k}$ and $k$ only.

We apply the above conclusion with $\left|z_{1}\right|<1$ and

$$
F(z)=\left(1-\left|z_{1}\right|\right)^{-k} f\left(z_{1}+\left(1-\left|z_{1}\right|\right) z\right)
$$


instead of $f(z)$. Then clearly $F$ belongs to $M_{k}$ or $A_{k}$ if $f$ does.

We deduce the following result.

Corollary 2. If $k$ is a positive integer and $f \in M_{k}$, then $1 / f$ satisfies the hypotheses and hence the conclusion of Theorem 1 and Corollary 1 with $\delta(r)=\frac{1}{3} \delta_{k}$ for $0<r<1$, and

$$
\begin{gathered}
\frac{8 \lambda(r)}{\delta(r)}=\frac{24}{\delta_{k}}\left\{\lambda_{k}+k \log \frac{1}{1-r}\right\}, \\
\lambda^{*}(t)=\frac{24}{\delta_{k}}\left\{\lambda_{k}+k \log 2+2 k \log \frac{1}{1-t}\right\},
\end{gathered}
$$

where $\delta_{k}$ and $\lambda_{k}$ are positive constants depending only on $k$. If $f$ is also analytic then $f$ satisfies the same conclusions.

We suppose that $\left|z_{1}\right| \leq\left|z_{2}\right|=r$ and that $f \in M_{k}$. We consider

$$
F(Z)=\frac{1}{\left(1-\left|z_{1}\right|\right)^{k}} f\left(z_{1}+\left(1-\left|z_{1}\right|\right) Z\right) .
$$

Then clearly $F(Z) \neq 0$ and $F^{(k)}(Z)=f^{(k)}\left(z_{1}+\left(1-\left|z_{1}\right|\right) Z\right) \neq 1$ when $Z \in \mathbb{D}$, so that $F \in M_{k}$. In particular if $Z_{1}=0$ and $Z_{2}=$ $\left(z_{2}-z_{1}\right) /\left(1-\left|z_{1}\right|\right)$ and

$$
\left|Z_{2}\right|<\delta_{k}
$$

we cannot have

$$
\left|F\left(Z_{j}\right)\right| \leq e^{-\lambda_{k}}, \quad\left|F\left(Z_{j^{\prime}}\right)\right| \geq e^{\lambda_{k}},
$$

where $\left(j, j^{\prime}\right)$ is a permutation of $(1,2)$.

Returning to $f$, we see that

$$
\left|f\left(z_{j}\right)\right|<e^{-\lambda_{k}}\left(1-\left|z_{1}\right|\right)^{k}, \quad \text { and }\left|f\left(z_{j^{\prime}}\right)\right|>e^{\lambda_{k}}\left(1-\left|z_{1}\right|\right)^{k}
$$

imply that

$$
\left|z_{j^{\prime}}-z_{j}\right|>\delta_{k}\left(1-\left|z_{1}\right|\right)
$$

i.e.,

$$
\frac{\left|z_{2}-z_{1}\right|}{1-\left|z_{1}\right|}>\delta_{k}
$$

Now if $\eta=\left|z_{2}-z_{1}\right| /\left(1-\left|z_{1}\right|\right) \leq 1$, we have

$$
\begin{aligned}
\left|1-\overline{z_{1}} z_{2}\right| & =\left|1-\overline{z_{1}} z_{1}+\overline{z_{1}} z_{1}-\overline{z_{1}} z_{2}\right| \leq 1-\left|z_{1}\right|^{2}+\left|z_{2}-z_{1}\right| \\
& =\left(1-\left|z_{1}\right|\right)\left(1+\left|z_{1}\right|+\eta\right)<3\left(1-\left|z_{1}\right|\right) .
\end{aligned}
$$


Thus if $\left|z_{2}-z_{1}\right| \leq 1-\left|z_{1}\right|$, we have

$$
\left|\frac{z_{2}-z_{1}}{1-\overline{z_{1}} z_{2}}\right|>\frac{\left|z_{2}-z_{1}\right|}{3\left(1-\left|z_{1}\right|\right)}>\frac{\delta_{k}}{3}
$$

if (29) holds. We have assumed that $\left|z_{2}-z_{1}\right| \leq 1-\left|z_{1}\right|$. But if this is false, (30) is still true by the maximum principle. Thus (28) always implies (30), and so does

$$
\left|f\left(z_{j}\right)\right|<(1-r)^{k} e^{-\lambda_{k}}, \quad\left|f\left(z_{j^{\prime}}\right)\right|>(1-r)^{-k} e^{\lambda_{k}}
$$

since this implies (27) and hence (28), because $r=\max \left\{\left|z_{j}\right|,\left|z_{j^{\prime}}\right|\right\}$. This proves Corollary 2.

We note that the hypotheses (1) to (3) in Theorem 1 are the same for $f$ and $1 / f$. Hence if $f$ satisfies (1) to (3) and $1 / f$ is analytic, then $1 / f$ satisfies the conclusions of Theorem 1. In particular if $f \in M_{k}$, so that $f \neq 0$ in $\mathbb{D}$, we can apply the conclusion of Theorem 1 to $1 / f$ instead of $f$, with $\lambda(r)$ given by (23) and hence $\lambda^{*}(r)$ by (24).

As another example we have the following result. We denote by $\mathcal{M}_{k}$ and $\mathcal{A}_{k}$ respectively the family of meromorphic functions $f$ and analytic functions $f$ in $\mathbb{D}$ such that $f^{\prime}(z) f(z)^{k} \neq 1$ in $\mathbb{D}$. By [3, Theorem 4.4.18, p. 132], the family $\mathcal{A}_{k}$ is normal. For the result that also the family $\mathcal{M}_{k}$ is normal we refer the reader to $[4$, p. 226] and the references given therein.

Corollary 3. If $k$ is a positive integer and $f \in \mathcal{A}_{k}$, then $f$ satisfies the hypotheses and hence the conclusion of Theorem 1 and Corollary 1 with $\delta(r)=\eta_{k}$, and

$$
\lambda(r)=\mu_{k}+\frac{1}{k+1} \log \frac{1}{1-r}
$$

where $\eta_{k}$ and $\mu_{k}$ are positive constants depending only on $k$.

We write $F(z)=\left(1-\left|z_{1}\right|\right)^{-1 /(k+1)} f\left(z_{1}+\left(1-\left|z_{1}\right|\right) z\right)$ and proceed as in the proof of Corollary 2.

The functions in $M_{k}$ and those in $\mathcal{M}_{k}$ also have locally bounded characteristic. This follows directly from Theorem 1 and Theorem 3 of $[2]$. In fact if

$$
\mu(r)=\sup _{|z| \leq r} f^{\sharp}(z)=\sup _{|z| \leq r} \frac{\left|f^{\prime}(z)\right|}{1+|f(z)|^{2}},
$$

then even for meromorphic $f$ we have under the hypotheses of Corollaries 2 and 3 that

$$
\mu(r)=O\left(\frac{1}{1-r}\right) \log \frac{1}{1-r},
$$


so that

$$
\int_{0}^{1}(1-r)^{1 / 2} \mu(r) d r<\infty
$$

which is the hypothesis of Theorem 3 in [2].

For the definition of locally bounded characteristic and other applications we refer the reader to [2].

\section{REFERENCES}

[1] W.K. Hayman, Uniformly normal families, pp. 199-212 in Lectures on functions of a complex variable, The University of Michigan Press, Ann Arbor, 1955.

[2] W.K. Hayman and Ch. Pommerenke, On meromorphic functions with growth conditions, pp. 61-80 in Complex Analysis, Birkhäuser, Basel, 1988.

[3] Joel Schiff, Normal families, Springer, New York, 1993.

[4] Lawrence Zalcman, Normal families: new perspectives, Bull. Amer. Math. Soc. (N.S.) 35 (1998), 215-230.

Department of Mathematics, Imperial College, London SW7 2BZ, U.K.

University of Illinois at Urbana-Champaign, Department of Mathematics, 1409 West Green Street, Urbana, IL 61801 USA

E-mail address: aimo@uiuc.edu 